\title{
RADIO INTERFEROMETRY OF VENUS AT SHORT
}

\section{WAVELENGTHS}

\author{
MICHAEL A. JANSSEN \\ Jet Propulsion Laboratory, California Institute of Technology, \\ Pasadena, Calif., U.S.A.
}

\begin{abstract}
From short wavelength $(\lambda<4 \mathrm{~cm})$ interferometry of Venus interpreted on the basis of the physical structure and bulk composition of its atmosphere as given by spacecraft measurements, evidence for the presence of a source of opacity in addition to $\mathrm{CO}_{2}$ is founa. This opacity presumably is due to unknown minor constituents of the atmosphere of Venus.
\end{abstract}

\section{Introduction}

The source of the microwave opacity of the atmosphere of Venus is strictly unknown. Pressure-induced absorption by the bulk constituent $\mathrm{CO}_{2}$ can explain many features of the emission observed at radio wavelengths. It is likely, however, that additional microwave absorption is present beneath the visible cloud layer. Trace amounts of strongly polar molecules such as $\mathrm{H}_{2} \mathrm{O}$ and $\mathrm{NH}_{3}$ can contribute measurably to the opacity. In concentrations sufficient to affect the microwave emission such molecules can have important implications for the thermal and chemical properties of the atmosphere. The question of the source of the microwave opacity is therefore an interesting one to pursue.

The identification of this source cannot be made uniquely since no molecular resonances are observed. Rather, the radio observations must be compared with predictions made by an emission model based on independent evidence. The composition and physical structure of the atmosphere are known from the Mariner and Venera spacecraft experiments (Fjeldbo et al., 1971; Avduevsky et al., 1970, 1971). The microwave properties of the surface may be deduced from the radar cross-section measurements (Muhleman, 1969). Using the absorption coefficient of $\mathrm{CO}_{2}$ found from laboratory measurements (Ho et al., 1966) one may specify a detailed model for the microwave emission. The presence of additional absorption may be inferred from differences between the predictions of this model and the observed emission. Pollack and Morrison (1970) have examined the radar and disk temperature measurements on this basis and find a mixing ratio of water vapor in the range $0.3 \%$ to $1.0 \%$ to be compatible with the existing data. A more recent series of measurements in the neighborhood of the $\mathrm{H}_{2} \mathrm{O} \lambda 1.35 \mathrm{~cm}$ resonance shows no evidence for water vapor to an upper limit of $0.2 \%$ for the mixing ratio, and generally limits the absorption which may be present in the uppermost region of the atmosphere beneath the visible cloud deck (Janssen et al., 1973; Janssen, 1973). From the frequency dependence of the radar cross-section Muhleman finds the total opacity of the atmosphere to be consistent with the law $\tau=(14.6 \pm 0.6) / \lambda^{2}$, where $\lambda$ is the wavelength in centimeters. Sinclair et al. (1972) interpret their interferometric measurements at $\lambda 11 \mathrm{~cm}$ to be consistent 
with a larger opacity $\tau=22.7 / \lambda^{2}$. For microwave absorption by $\mathrm{CO}_{2}$ alone, the model calculation described below predicts $\tau=11 / \lambda^{2}$. Berge et al. (1972) find no simple model for the surface and atmosphere which is fully consistent with their interferometric measurements in the wavelength range $3-21 \mathrm{~cm}$. A larger opacity than given by $\mathrm{CO}_{2}$ alone is suggested, however.

We consider here radio interferometric observations at wavelengths less than $\lambda 4 \mathrm{~cm}$ The atmospheric emission dominates the surface contribution at these wavelengths, and observations in this region of the spectrum are particularly appropriate for the study of the atmospheric absorption. Here the frequency dependence of the emission contains information both on the total atmospheric opacity and the distribution of absorption with altitude. Further, uncertainties about the surface contribution to the emission are of less significance. Interferometric measurements may be made with great precision. Although the interpretation of interferometric data is less straightforward than for disk temperature or radar cross-section measurements it is demonstrated here that it is possible to discriminate more clearly among plausible cases for the absorption. The few existing measurements examined here give evidence for the presence of a source of opacity in addition to $\mathrm{CO}_{2}$, with some indication about its distribution with altitude. The rapid development of the technique of interferometry at millimeter wavelengths will permit more conclusive measurements to be made in the future.

\section{Discussion}

The response of an ideal two-element interferometer to an extended source of emission is called the 'visibility function'. The argument of this function is the baseline, in wavelengths, as projected onto the sky in the direction of the source. This describes the 'spatial frequency' of the finely spaced interferometer fringes on the plane of the sky, and for present purposes it is convenient to normalize this spatial frequency to the variable planet radius as

$$
\beta=\frac{\text { ephemeris semi-diameter of Venus }}{\text { angular fringe spacing }} \text {. }
$$

The visibility function is related to the brightness distribution of the source through a Fourier transform. In principle, if data is obtained for a sufficiently extensive sampling of spatial frequencies (baseline separations) a detailed map of the source in spatial coordinates may be obtained. In practice this is difficult to achieve. Further, it is largely unnecessary since many important questions may be answered by a very restricted sample of the data.

We consider here two particular measurements; that of the first zero of the unpolarized visibility function $\beta_{1}$, and of the polarized visibility $V_{\perp}-V_{\|}$in the neighborhood of this zero. The quantity $\beta_{1}$ is proportional to the baseline separation at which the source is just resolved, and depends upon the overall size of the source. In the case of Venus the boundaries of the emission are well known, while the distribution of brightness across the disk is not. In effect $\beta_{1}$ gives a measure of the overall limb dark- 
ening, which in turn depends upon the temperature lapse rate of the atmosphere, the distribution of absorption with altitude, and the surface dielectric constant. We define the polarized visibility $V_{\perp}-V_{\|}$as the difference between the visibilities with the linear polarizations at the two antennas perpendicular and parallel respectively to the projected baseline. Since the surface contribution is polarized while the atmospheric contribution is not, the polarized visibility can be interpreted to give the total opacity of the atmosphere. Both quantities can be precisely measured. The determination of the first zero $\beta_{1}$ is effectively a null measurement, and is limited in practice by the signal-to-noise ratio. The polarized visibility in the neighborhood of $\beta_{1}$ is uncontaminated by instrumental polarization errors.

The existing measurements of the first visibility zero are shown in Figure 1. The error bars depict one standard deviation. The $\lambda 1.35 \mathrm{~cm}$ point combines results from the 1970 and 1972 conjunctions reported by Janssen et al. (1973) and Janssen (1973). The point at $\lambda 3.1 \mathrm{~cm}$ was communicated by Berge (1973), and is based on observations described in Berge and Greisen (1969). The $\lambda 3.7 \mathrm{~cm}$ point was determined from data taken by E. T. Olsen during the 1972 inferior conjunction with the National

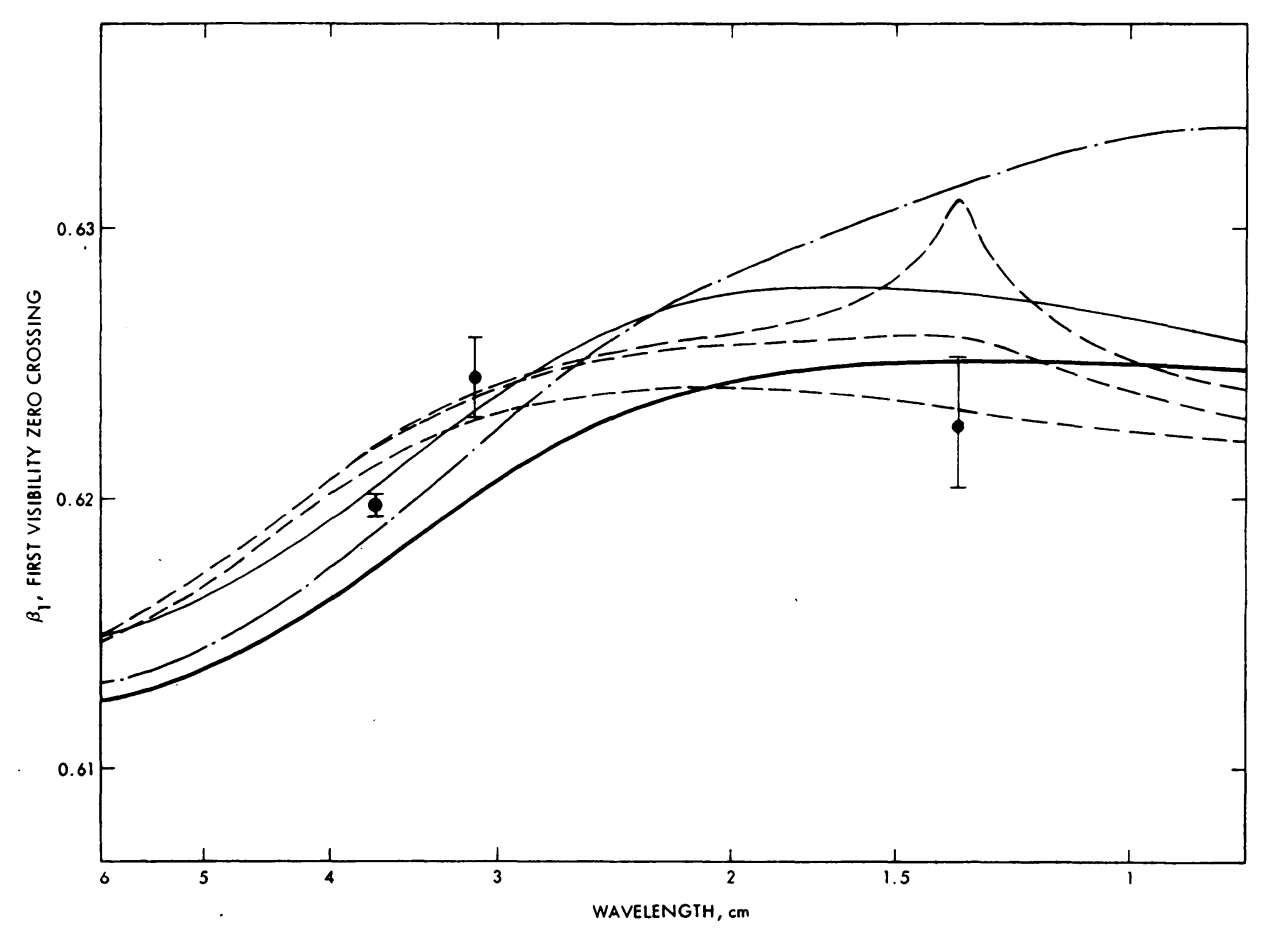

Fig. 1. Limb darkening of Venus in the wavelength range $0.8-6 \mathrm{~cm}$ as measured by the first zero crossing of the visibility function. The heavy solid curve is calculated for a model in which the only source of microwave opacity is atmospheric $\mathrm{CO}_{2}$. The remaining curves were calculated for models to include the effect of additional absorption by water (dashed curves), a constant absorber between $35 \mathrm{~km}$ and $50 \mathrm{~km}$ altitude (light solid curve), and an absorber at $60 \mathrm{~km}$ (dot-dash curve) according to details in the text. The points are from existing observations shortward of $\lambda 4 \mathrm{~cm}$. 
Radio Astronomy Observatory three-element interferometer (Olsen, 1973). Two days of data were obtained, which included two first zero crossings each day with the baseline projections approximately east-west and north-south respectively. The determination of $\beta_{1}$ was made by averaging all data in the interval $0.59<\beta<0.63$, with the resulting small formal error. These observations are presently being examined in more detail by Olsen.

A computer program was written to calculate the visibility function and disk temperature of Venus for several different cases for the microwave absorption. In the computation a thermal, non-scattering transfer of radiation is assumed. Geometric effects such as the finite thickness of the atmosphere and refraction of the emergent rays are taken into account. The surface is assumed to be smooth and horizontally uniform with a dielectric constant $\varepsilon=4.8$. The atmospheric profile is based on measurements obtained by the Venera 4-7 atmospheric probes (Avduevsky et al., 1970, 1971). The program makes use of the pressures and temperatures listed in Table I, inter-

TABLE I

Basic atmospheric model

\begin{tabular}{lll}
\hline $\begin{array}{l}\text { Altitude } \\
(\mathrm{km})\end{array}$ & $\begin{array}{l}\text { Pressure } \\
(\mathrm{atm})\end{array}$ & $\begin{array}{l}\text { Temperature } \\
(\mathrm{K})\end{array}$ \\
\hline 76 & 0.008 & 195 \\
64 & 0.1 & 243 \\
57 & 0.33 & 275 \\
51 & 0.86 & 312 \\
45.9 & 1.7 & 355 \\
37.3 & 4.8 & 432 \\
28.5 & 11.5 & 515 \\
19.6 & 24.2 & 588 \\
9 & 53.1 & 676 \\
0 & 96.0 & 747
\end{tabular}

polating between points in the table using an isentropic expansion with a constant temperature lapse rate. The bulk composition of the atmosphere is assumed to be $95 \% \mathrm{CO}_{2}$ and $5 \% \mathrm{~N}_{2}$.

The heavy solid curve of Figure 1 was calculated for this basic model, assuming $\mathrm{CO}_{2}$ to be the only source of microwave opacity. The $\lambda 1.35 \mathrm{~cm}$ point is consistent with this curve; however, the measurements of $\beta_{1}$ at $\lambda 3.1$ and $\lambda 3.7$ are significantly greater than the basic model predicts. The remaining curves correspond to calculations for particular cases, described below, in which other absorbers are added to this model. The intent of these cases is primarily to examine the behavior of the visibility for plausible absorption models, rather than to search for a particular explanation for the present data.

A uniformly bright disk of the radius of Venus would yield a horizontally straight line at $\beta_{1} \approx 0.61$ in Figure 1 . The model curves show differing amounts of limb darkening with a strong wavelength dependence. Note that the solid curve of the basic 
model shows a generally wavelength-independent behavior shortward of $\lambda 2 \mathrm{~cm}$. In this region the emission originates in the atmosphere and the limb darkening is controlled by the approximately constant lapse rate. At longer wavelengths there is a tendency towards limb brightening, i.e., $\beta_{1}$ tends to smaller values, as the surface begins to become visible near the sub-Earth point. The surface, at the same temperature as the adjacent atmosphere, appears somewhat cooler due to its reduced emissivity.

The data indicate an increase in limb darkening over the basic model at the longer wavelengths considered here. The addition of opacity to the model generally increases the limb darkening longward of approximately $\lambda 2 \mathrm{~cm}$, while the exact wavelength dependence is sensitive to the way in which the additional opacity is distributed with altitude. The polarization data impose a constraint on the total opacity. We first consider the simple case in which the absorption of the basic model is uniformly increased by a factor $f$. The resulting emission is in every way identical after the wavelength transformation $\lambda \rightarrow f^{1 / 2} \lambda$, which follows from the $\lambda^{-2}$ dependence of the $\mathrm{CO}_{2}$ absorption coefficient. By transposing the heavy solid curve of Figure 1 to fit the data, we find $f=1.5$.

The lighter curves of Figure 1 illustrate the effect produced by several other plausible models for the additional absorption. The three dashed curves show the effect of adding water vapor to the atmosphere. In the uppermost curve water vapor is added at a constant mixing ratio of $0.5 \%$ by volume up to the cloud top level at $60 \mathrm{~km}$ altitude. The non-resonant absorption increases the limb darkening for $\lambda>2 \mathrm{~cm}$, and would provide a best fit to the data for a mixing ratio of $0.3 \%$. The $\lambda 1.35 \mathrm{~cm}$ point is in disagreement with the limb darkening produced by the $\mathrm{H}_{2} \mathrm{O}$ resonance, however, and yields an upper limit for this case of $0.2 \%$ for the mixing ratio. If water vapor is removed from the upper layers of the atmosphere where the $\lambda 1.35 \mathrm{~cm}$ line is produced, a more consistent fit to the data is possible. The lower two dashed curves were generated from model calculations in which the $0.5 \%$ mixing ratio was arbitrarily set to zero above $50 \mathrm{~km}$ and $40 \mathrm{~km}$, respectively. Such a drying out of the upper atmosphere is not consistent with simple water vapor saturation, but is possible with chemical reactions: The affinity of $\mathrm{H}_{2} \mathrm{SO}_{4}$ for water can produce such an effect, for example (Young, 1973).

The addition of absorption at very high altitudes produces a generally poor fit to the data. The dot-dash curve of Figure 1 shows a case in which a thin layer of optical depth $\tau=0.2 / \lambda^{2}$ is placed at $60 \mathrm{~km}$ altitude. The $\lambda^{-2}$ dependence would approximate that for small liquid water droplets. A large increase in limb darkening is produced at short wavelengths, while little effect is apparent beyond $\lambda 3 \mathrm{~cm}$. A source of opacity at intermediate altitudes can provide a more acceptable fit. The light solid curve was calculated for the case of a constant absorption $\alpha=0.012 / \lambda \mathrm{km}^{-1}$ between $35 \mathrm{~km}$ and $50 \mathrm{~km}$ altitude. This case is consistent with the anomalous attenuation found from the Mariner V occultation experiments (Fjeldbo et al., 1970) if the attenuation is produced by an absorption varying with wavelength to the inverse first power. The atmosphere below $35 \mathrm{~km}$ altitude was not measured in the occultation experiments, and the lower altitude cut-off in absorption was arbitrarily assumed for this case. 
Figure 2 shows the polarized visibility $V_{\perp}-V_{\|}$measured at $\lambda 3.7$ and $\lambda 3.1 \mathrm{~cm}$ along with calculations for the models considered above. The polarized visibility is sensitive only to the total opacity. The polarized visibility measured at $\lambda 3.7 \mathrm{~cm}$ is about twothirds of that predicted by the basic model (heavy solid curve), implying an actual increase in opacity over the basic model by a factor of 1.2. This may be compared with the factor $f=1.5$ found above. While absorption at high altitudes has a strong effect on the limb darkening, there is little effect on the total opacity. The case of absorption at $60 \mathrm{~km}$ altitude coincides with the heavy solid curve of Figure 2. A slight

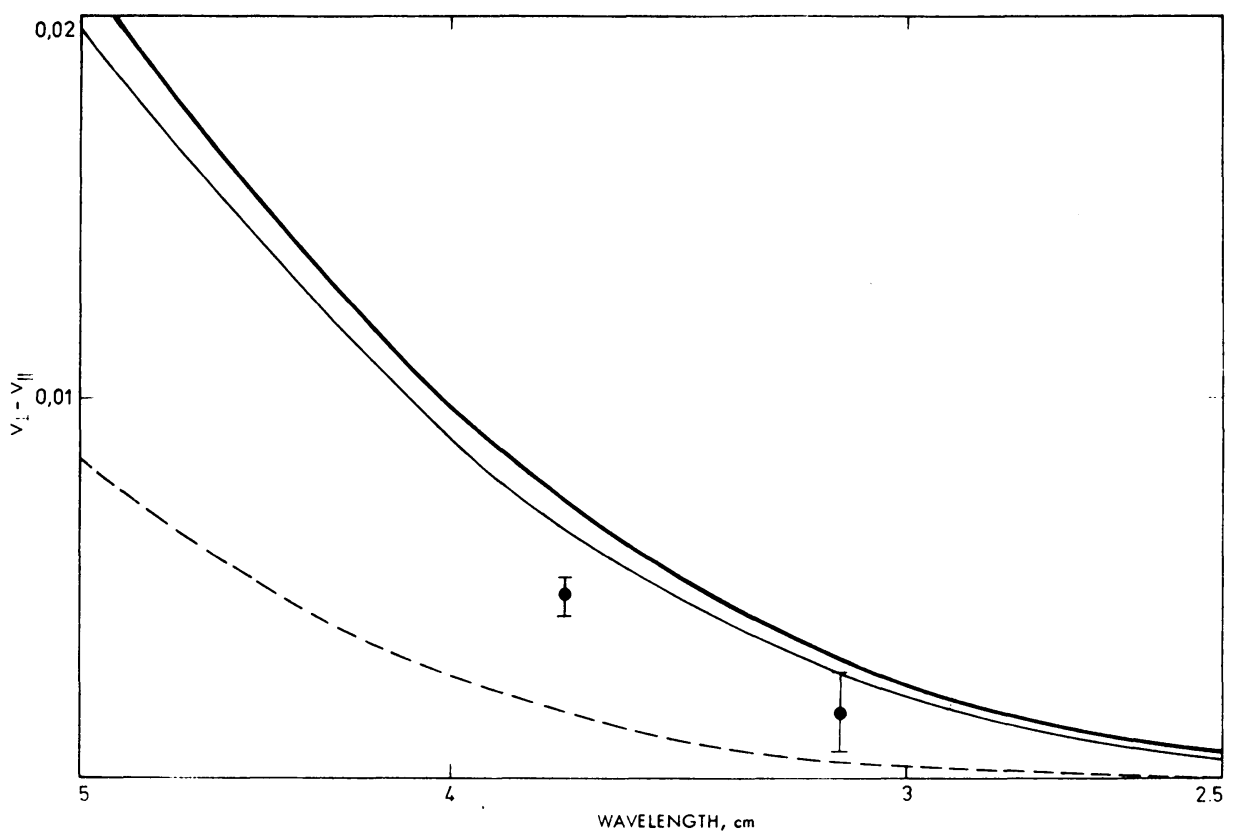

Fig. 2. The polarized visibility of Venus in the neighborhood of $\beta=0.61$. The curves correspond to the cases considered in Figure 1.

decrease in total opacity is seen for the case of an absorber at intermediate altitudes, as shown by the light solid curve. The models with water vapor, which coincide to give the single dashed curve of Figure 2, are seen to produce far too much opacity. The $\lambda 3.7 \mathrm{~cm}$ measurement calls for a mixing ratio of only $0.15 \%$.

These inferences depend on the credibility of the basic model used in the calculations. Several features of this model may be altered to provide an explanation of the interferometric data without invoking an additional source of opacity. For example, a computation was made in which the surface dielectric constant was reduced from 4.8 to 2.5. Although in disagreement with the radar data, this provided an acceptable fit to both the limb darkening and polarization data. Also, if the $\mathrm{CO}_{2}$ absorption coefficient is incorrect the error would have to be as large as $20 \%$ or $50 \%$ from the above determinations. We may further consider systematic errors in the spacecraft 
measurements. Increasing the $\mathrm{CO}_{2}$ content from $95 \%$ to $100 \%$ gives a $10 \%$ increase in opacity. The $\mathrm{CO}_{2}$ absorption coefficient is proportional to $P^{2} / T^{5}$; the limb darkening data would require a reduction in temperature by nearly $50 \mathrm{~K}$ throughout the atmosphere, or an increase of pressures by a factor of 1.2 , to increase the absorption coefficient of $\mathrm{CO}_{2}$ to the required value. We note that where they overlap, temperatures from the $S$-band occultation measurement are about $20 \mathrm{~K}$ higher than the corresponding Venera measurements. Finally, upon lowering the mean surface of the model by $4 \mathrm{~km}$, the required increase in opacity is provided by the extra layer of atmosphere. The surface temperature and pressure would be raised by $30 \mathrm{~K}$ and $25 \mathrm{~atm}$, respectively, as a result. None of these choices appear consistent with present knowledge.

Disk temperature spectra calculated for the above models are shown in Figure 3. Except for the case in which water vapor extends to $60 \mathrm{~km}$ altitude, there is a relative lack of distinguishing structure among the cases considered. Also, not apparent in the figure is the increased sensitivity to model parameters. If atmospheric composition and temperature are varied within the uncertainty permitted by the spacecraft measurements, a range in disk temperature of more than $5 \%$ is possible at a given wavelength. This is sufficient to obliterate differences between all but the most extreme

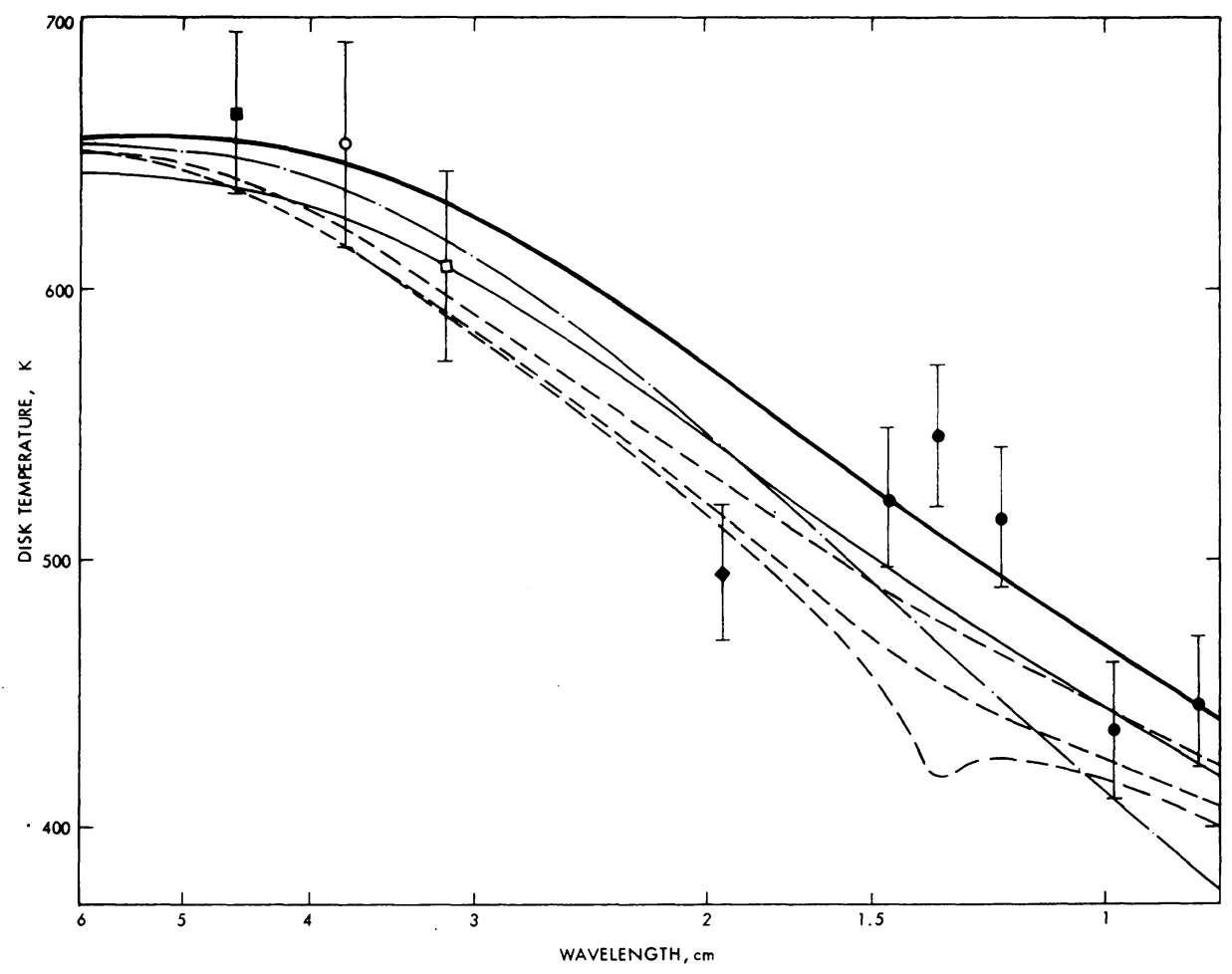

Fig. 3. The disk temperature spectrum of Venus at short wavelengths. The curves correspond to the cases considered in Figure 1. The data points are from Warnock and Dickel, 1972, $\square ;$ Klein, 1973, : Berge et al., 197 $\square$ : Pollack and Morrison, 1970, $\diamond$; Janssen et al., 1973, 
cases of Figure 3. The data points represent a sampling of recent measurements in this wavelength range. The error bars indicate the expected standard deviations of the measurements and primarily reflect the uncertainties of the absolute flux calibrations. These uncertainties amount to at least $5 \%$. Again, all but the more extreme cases are in acceptable agreement with the measurements. In contrast to the potential of millimeter wavelength interferometry, the flux calibration at these wavelengths is not likely to be greatly improved in the near future.

\section{Conclusions}

On the basis of present knowledge of the atmosphere and surface of Venus, the interferometric measurements at wavelengths less than $\lambda 4 \mathrm{~cm}$ reveal the presence of a source of microwave opacity in addition to $\mathrm{CO}_{2}$. If $\mathrm{CO}_{2}$ were indeed the only absorber the measurements would imply significant errors in either the spacecraft measurements of the atmospheric structure, the interpretation of the radar measurements, or the laboratory measurements of the $\mathrm{CO}_{2}$ absorption coefficient. The limb darkening, as measured from the first zero of the visibility function at $\lambda 3.1$ and $\lambda 3.7 \mathrm{~cm}$, indicates an amount of additional absorption equivalent to increasing the $\mathrm{CO}_{2}$ absorption by a factor of 1.5 in the basic model given by spacecraft, radar, and laboratory measurements. The polarized visibility at $\lambda 3.7 \mathrm{~cm}$ is consistent with an increase in the total opacity by a factor of 1.2 over the case for $\mathrm{CO}_{2}$ absorption alone. These amounts are consistent with Muhleman's interpretation of the radar data giving an increase in opacity by a factor of 1.35 , but less than that found by Pollack and Morrison and by Sinclair et al.

The apparent discrepancy between the opacity found from the limb darkening measurements and from the polarization are easily resolved if we consider possible variations in the absorption with altitude. Absorption at or near the cloud top level is ruled out by the limb darkening at $\lambda 1.35 \mathrm{~cm}$. Strong absorption near the surface would mostly affect the total opacity, thereby increasing the discrepancy. A concentration of absorption at intermediate levels relative to $\mathrm{CO}_{2}$ is preferred, although it is difficult to draw definite conclusions because of present data and model uncertainties. Particular cases such as water vapor at concentrations in the range $0.15-0.3 \%$ or an absorber at intermediate altitudes provide acceptable agreement with the observations. Water vapor is, of course, not specifically preferred over other non-resonant molecular absorbers. If the absorber at intermediate levels is to be identified with the source of attenuation of the $S$-band occultation signal, however, a dependence of the absorption approximately on the inverse first power of the wavelength is required. This would imply a particulate, rather than a molecular absorber.

\section{Acknowledgements}

I wish to thank Dr E. T. Olsen of the Jet Propulsion Laboratory for making available his interferometric data on Venus and several useful discussions about their inter- 
pretation. I would also like to thank D. O. Muhleman and G. L. Berge of the California Institute of Technology, for useful discussions and the use of their unpublished data. The present research was supported through a National Research Council Resident Research Associateship.

\section{References}

Avduevsky, V. S., Marov, M. Ya., and Rozhdestvensky, M. K.: 1960, J. Atmospheric Sci. 27, 561. Avduevsky, V. S., Marov, M. Ya., Rozhdestvensky, M. K., Borodin, N. F., and Kerzhanovich, V. V.: 1971, J. Atmospheric. Sci. 28, 263.

Berge, G. L. and Greisen, E. W.: 1969, Astrophys. J. 156, 1125.

Berge, G. L., Muhleman, D. O., and Orton, G. S.: 1972, Icarus 17, 675.

Berge, G. L.: 1973, private communication.

Fjeldbo, G., Kliore, A. J., and Eshleman, V. R.: 1971, Astron. J. 76, 123.

Ho, W., Kaufman, I. A., and Thaddeus, P.: 1966, J. Geophys. Res. 71, 5091.

Janssen, M. A.: 1973, Bull. Am. Astron. Soc. 5, 302.

Janssen, M. A., Hills, R. E., Thornton, D. D., and Welch, W. J.: 1973, Science 179, 994.

Klein, M. J.: 1973, private communication.

Muhleman, D. O.: 1969, Astron. J. 74, 57.

Olsen, E. T.: 1973, private communication.

Pollack, J. B. and Morrison, D.: 1970, Icarus 12, 376.

Sinclair, A. C. E., Basart, J. P., Buhl, D., and Gale, W. A.: 1972, Astrophys. J. 175, 555.

Warnock, W. W. and Dickel, J. R.: 1972, Icarus 17, 684.

Young, A. T.: 1973, Icarus 18, 564. 